\title{
A SURVEY ON SUPERCAPACITORS AND THEIR APPLICATIONS
}

\author{
Vandana Sharma ${ }^{1}$, Avesh Garg ${ }^{2}$, Suresh $\operatorname{Sood}^{3}$ \\ ${ }^{I}$ M. Tech Scholar, Electronics and Communication, ACE, Ambala, Haryana. India \\ ${ }^{2}$ Assistant Professor, Electronics and Communication, ACE, Ambala, Haryana, India \\ ${ }^{3}$ Research Associate, Electronics and Communication, ACE, Ambala, Haryana, India
}

\begin{abstract}
The need for more sustainable, capable energy storage has motivated a transformed scientific and commercial concern in advanced capacitor designs in which the suite of experimental techniques and ideas that comprise nanotechnology are playing a vital role. Capacitors might be charged and discharged speedily and are one of the primary building blocks of many types of electrical circuit, from microprocessors to large-scale power supplies, but generally have reasonably low energy storage potential when compared with batteries. The blende of nanorange materials with bespoke morphologies and properties to electrochemical supercapacitors is being intensively studied and observed .Recuperating and preserving energy of a moving vehicle as it slows down and accelerate the vehicle later by means of that preserved energy, can significantly increase fuel efficiencies of automobiles. Capacitors are one of the primary and basic building blocks of many types of electrical circuit. Therefore, an effort here is done to present a survey on supercapacitors.
\end{abstract}

Key Words: Super capacitors, EDLC, Grapheme, nanocomposites ****

\section{INTRODUCTION}

Batteries and capacitors have been used for energy storage till date. There is special class of device which has grab attention from last few years known as "Supercapacitor" or "Ultracapacitor". The most important energy storage devices "Supercapacitors" have been extensively used in number of requirements. When comparing batteries, regular capacitors and supercapacitors, it is noticed that the battery' $s$ take up to 3 hours from few seconds to charge, ordinary capacitor's take very less time approximately 1 million seconds while supercapacitor's take just 30 seconds for charging as well as discharging. Power density of supercapacitor is very high as compared to battery's and ordinary capacitor's power density. However there is still a need to enhance one important parameter energy density, which is low as compared to battery and normal capacitor. Moreover, batteries are manufactured from hazardous materials whereas supercapacitors are eco-friendly and more reliable. Supercapacitors are of three types that are electric double layer capacitor (EDLC), pseudocapacitor[1] and hybrid supercapacitors.

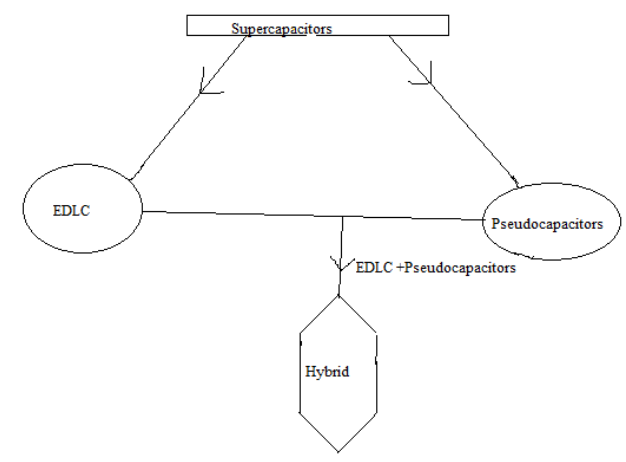

Fig-1. Types Of Supercapacitors
Hybrid ones are those which are combination of EDLCs and pseudocapacitors. EDLCs make use of the phenomenon of separation of charge inside a Helmholtz double layer to store electrostatic charge while pseudocapacitors utilize faradaic redox reactions with charge-transfer for the same storage purpose. Supercapacitors can use electrolytes either in liquid/gel or solid state.

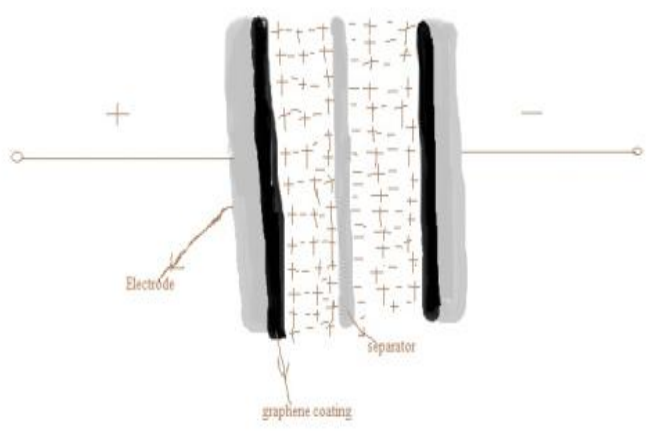

Fig-2. EDLC Showing Double Ion Layer Formation

There are certain parameters like high ionic conductivity, interfacial stability and mechanical stability which are quiet important consideration while choosing electrolyte in all the three forms. As mentioned above the supercapacitors are unlike ordinary capacitors as they utilize material which can enhance the surface area of electrodes. Out of these materials, graphene is proved to be the most attractive as it has a number of interesting properties like elevated electron and hole mobility, elevated surface area, zero band gap and carriers confinement[2] to a one atom thick layer. So EDLCs make use of graphene to increase the surface area of electrodes in order to store more charge, thereby increasing the capacitance finally. So, in this paper, an attempt has been done to provide a survey on supercapacitors along with there advantages/disadvantages and applications. 


\section{RELATED WORK}

The electric double-layer capacitor cause was first observed in 1957 by H.I. Becker of General Electric who was experimenting with devices using porous carbon electrodes. He stated that the energy was preserved in the carbon pores and it comprised "superbly high capacitance", while the was still inexplicable. The modern versions of the devices were developed by researchers at Standard Oil of Ohio in 1966, after by coincidence they again revealed the cause while functioning on investigational fuel cell designs. This cell design used two layers of activated charcoal separated by a thin porous insulator, and it remains the center point of electric double-layer capacitors . However, Standard Oil also failed to commercialize their invention but handover the technology license to NEC, which marketed the results as "supercapacitors" in 1978, to offer backup power for regulating computer memory. The market extended leisurely due to elevated costs, restricted performance and lack of awareness of the possibilities, but around the mid-1990s various advances in materials science and development of the system led to speedily modifying performance and an equally hasty reduction in cost. The ultracapacitor market in 2005 was between US $\$ 272$ million and $\$ 400$ million, depending on the source. Most of that money is spent by the automotive sector, where the devices are large and in unstandardized shapes. In 2008, Jayalakshmi $M$ and Balasubramanian K. [1] provided an overview of simply approaching supercapacitors for different needs. An overview of EDLC mechanism was described. . In 2010 the scientists from US were able to produce graphene nanowires by using thermochemical nanolithography. The researchers were able to produce supercapacitors through graphene by using a simple DVD Lightscribe writer on PC in past. The effectiveness of the supercapacitor is the important factor to note in mind. Years back, scientists were successful to form supercapacitors that were proficient enough to store 150 farads per gram, but they also adviced that the theoretical superior limit for graphene dependent supercapacitors is $\mathbf{5 5 0}$ $\mathrm{F} / \mathrm{g}$. This is mainly inspiring when compared against current technology: a commercially existing capacitor exhibit 1 Farad of electrostatic energy at 100 volts would be about $220 \mathrm{~mm}$ high and weigh about $2 \mathrm{kgs}$. Next in 2012, Mahapatra.S et al.[2] discussed the supercapacitor background, describing the Ferradic and non-ferradic processes of making capacitors and then discussed the role of nanomaterial for implementing supercapacitors. It states that by using nanorange materials the capacitance naturally got increased due to it's high surface to volume ratio and low matrix resistivity. Later in 2014, Shao Quingguo et al.[3] described the hydrothermal method for preparing carbon nanotube spaced graphene aerogels. It is mention- ed that the composite aerogels convey a $33 \%$ larger specific capacitance of $183.3 \mathrm{~F} / \mathrm{g}$ at $0.5 \mathrm{~A} / \mathrm{g}$ and a high energy density of $80 \mathrm{WH} / \mathrm{Kg}$ when using an ionic liquid as the electrolyte. Later on, Ban Shuai et al.[4] discussed graphene based supercapacitor using a multidisciplinary approach and discussed that the capacitance of graphene is limited by internal resistance and mass transfer. Guzman et al. explained the effects of graphene and $\mathrm{C}$ coating modifications on electrochemical act of silicon nanoparticle/graphene composite anode. Moreover, optimization of the methods to achieve the best alteratered characteristics might enable performance modifications that exploit the capabilities of the materials. Then Zheng Chao et al.[5] introduced the electrochemical capacitor, porous graphene/activated carbon preparation via hydrothermal carbonization and two-step chemical activation. The composites owe a high packing density and has a specific capacitance of $210 \mathrm{~F} / \mathrm{g}$. It is mentioned that a high capacity preservation rate of $94.7 \%$ after 50000 can be achieved. It is concluded that, the nanosheet like electrode material has a short diffusion pathway, which helps the rapid transport of the electrolyte ions. In the same year, Zhou Yu et al. [6] discussed the graphene/carbon black hybrid films preparation by a simple vacuum filtration method. The supercapacitor shows wonderful rectangular shape even at high scan rate of $5 \mathrm{~V} / \mathrm{s}$. The supercapacitor remained $61.3 \%$ capacitance when the scan rate increased from 0.005 to $5 \mathrm{~V} / \mathrm{s}$. The solid state flexible supercapacitor shows excellent rate performance and cycling stability. After that Bello Abdulhaleem et al. [7] described the three dimensional synthesis of graphene foam (GF) using CVD. It explains the process of microwave treatment of graphene foam with PVA and formaldehyde to produce 3D hydrogel, production of porous carbons by $\mathrm{KOH}$ activation of the hydrogel.. Also, Jin Hong et al. [8] provided the nitrogen-doped graphene(Ndoped graphene) details produced from dried distillers grains with soluble DDGS which show high specific capacitance. Then, the SEM and TEM results are demonstrated. In the same context, Cai Xiaoyi et al.[9] discussed the growth of $\mathrm{CO}(\mathrm{OH})_{2}$ nanowires on $\mathrm{N}$ modified graphene(NMEG) surface and its characterization is discussed. Moreover, it is verified that the large voltage window characteristic of polypyrrole graphene composite(PPy/rG-o) and the specific energy of $24.9 \mathrm{Wh} / \mathrm{Kg}$ shown by $\mathrm{PPy} / \mathrm{rG}-\mathrm{O} / / \mathrm{CO}(\mathrm{OH})_{2} / \mathrm{NMEG}$ supercapacitor. Bo et al. [10] reported a one step fabrication method for supercapacitors (EDLC), with graphene grown on metallic current collector. Both aqueous and organic electrolytes were used to study the behavior of resulting electrode. It was concluded that vertical orientation compared with horizontal orientation of graphene sheets could lead to better specific capacitance. Du and Pan [11] proposed the use of carbon nanotubes as a promising material for supercapacitors. It described the advantages of increased power density.A number of methods for constructing supercapacitors using nanomaterials were introduced. Raj M P et al. [12] stated that ceramic-polymer composites can provide capacitance within $10 \mathrm{nF} / \mathrm{cm}^{2}$. Supercapacitor comprising nanostructured electrodes for high surface area per unit volume results in ultrahigh capacitance densities of the orders of hundreds of microfarad. Complete electrical characterization of carbon black-epoxy nanocomposites was reported. It stated that the high dielectric constant of 1000 at low frequencies can be achieved through carbon black epoxy composite. The high frequency $(0.5-4.5 \mathrm{GHz})$ dielectric constant was found to be only upto 10 times of polymer matrix. Gnanakan Prabhu R S et al. [13] proposed the synthesis of polythiophene(PTh) nanoparticles by the method of cationic surfactant assisted dilute polymerization using $\mathrm{FeCl}_{3}$ as oxidant for redox supercapacitor. PTh 
nanoparticles were used as electrode material. Then FT-IR, UV-visible spectroscopy, XRD and SEM techniques were used for the physical characterization of the synthesized PTh nanoparticles. The specific capacitance of capacitor was found to be $134 \mathrm{~F} / \mathrm{g}$. The energy and power densities were observed as $8 \mathrm{Whk} / \mathrm{g}$ and $396 \mathrm{WK} / \mathrm{g}$. Zhang $\mathrm{F}$ et al. [14] described a strategy for the preparation of $\mathrm{CO}_{3} \mathrm{O}_{4}$ nano/micro superstructures using solvothermal method, cobalt ion involving coordination polymer I association with metal-organic framework was synthesized by using $\mathrm{p}$ benzenedicarboxylic acid ( $\mathrm{p}-\mathrm{H}_{2} \mathrm{BDC}$ ) as ligand. By solidstate annealing of MOF precursor at $450^{\circ} \mathrm{C}$ for $2 \mathrm{~h}$, porous $\mathrm{CO}_{3} \mathrm{O}_{4}$ nano/micro superstructures were prepared. $\mathrm{CO}_{3} \mathrm{O}_{4}$ superpatterned structures possessed a specific capacitance of $208 \mathrm{~F} / \mathrm{g}$ at the current density of $1 \mathrm{~A} / \mathrm{g}$ and a specific capacitance of $97 \%$ after 1000 continuous charge-discharge cycles in $6 \mathrm{M}$ aq. $\mathrm{KOH}$ solution. Phillips Jonathan et al. [15] provided the details about super dielectric materials. High surface area alumina powders with a solution of boric acid or sodium chloride in water have very high dielectric constants of the range greater than $4 \times 10^{8}$.NPS capacitors with typical electrostatic capacitive behavior like increasing capacitance with decreasing thickness are discussed. The charging discharging graphs and capacitance-inverse thickness graphs are also discussed. Finally the high dielectric constants of NPS capacitors are claimed to surpass supercapacitors.

\section{ISSUES AND CHALLENGES}

Energy storage densities of ultracapacitors are currently inferior to batteries and are unsatisfactory for a number of applications. The need of hour is to produce high quality graphene in order to increase capacitance along with increasing the stability of electrolytes used is alarming point. Charge transfer and transport process is to be understood properly. The deployment of nanorange electrodes with improved, high surface area characteristic offers the potential for storing a number of charges, expanding charge density. By use of carbon nanotube, aerogels, drastically improved surface area and energy densities can be obtained. Graphene is one of the most promising materials for Ultracapacitor electrodes with prospect of power densities breaking record of any other known form of activated Carbon electrodes due to its large and readily accessible surface area. It means a compariatively larger number of ions in the electrolyte forms a layer on the graphene sheets resulting in extraordinary levels of stored charge. Graphene's ultra thin structure will allow for sheets of the material to be laid on one another to increase energy storage and possibly double the current capacity of Ultracapacitors. This would give permission to Ultracapacitors so that they expand into many other renewable and clean energy storage applications. However the main efforts which could be done to get better device characteristics are as follows

- Energy storage densities are presently inferior than they are for batteries and are insufficient for many applications.
- The diminutive but considerable amount of energy leftovers during discharge

- Voltage balancing is mandatory when using more than three capacitors in series.

- $\mathrm{C}=\varepsilon^{*} \mathrm{~A} / \mathrm{D}$

- $\quad$ So by increasing surface area using a porous material energy storage can be improved.

- Employing a dielectric material like $\mathrm{BaTiO}_{3}$ which has high dielectric constant equal to 4000 or more, high capacitance can be achieved.

- Using Super Dielectric Material, Supercapacitors with high energy density and high capacitance can be achieved.

\section{MERITS and DEMERITS}

The major advantages of using supercapacitor against batteries include extended life time, amplified rated voltage, wide range of working temperature, superior energy and power densities, excellent cyclability ( hundred of thousands charge/discharge cycles as compared with hundreds of cycles for storage batteries).[1] But like every device's limitation, supercapacitors too have some demerits like low energy density for example it generally holds $1 / 5^{\text {th }}$ to $1 / 10$ th of a battery. Inability to use the full energy spectrum for some applications. To get higher voltages, serial connections are required as these are low voltage cells. A voltage balancing element is always required to connect more than 3 supercapacitors in series to use accordingly for any application. Disadvantage of self-discharge in compared to electrochemical batteries.

\section{APPLICATIONS}

Electrochemical supercapacitors are still fairly novel devices that have yet to practice widespread use. This is all because of their restricted power and energy capabilities, and therefore find only useful in low power and energy applications like memory backup. Currently, great advances have been made in improving characteristics like energy and power density. Novel applications for EDLCs are being discover and promoting at excellent rate.[11] The supercapacitors find application in electric vehicles, battery enhancement, memory backup, improved quality of power and many more.

\section{FUTURE SCOPE AND CONCLUSION}

Keeping consideration of various advantages and use of supercapacitors for various applications, mentioned as above, the technology promises a bright future. Nanocomposites and nanoparticles increasing chase for research has brought many improvements in the device already. Further enhancement in the field of nanotechnology and improvement in graphene synthesis can lead to the 
elimination of the disadvantages and improvement in the various parameters like power density and energy density.

\section{ACKNOWLEDGEMENT}

The authors are thankful to Dr. C C Tripathi and Mr. Randhir from Kurukshetra University for providing valuable suggestion. Also authors would like to acknowledge the Management authorities and Dr. Amit Wason (Principal) of ACE, Devsthali, Ambala College for their help and support in implementing the work.

\section{REFERENCES}

[1] Jayalakshmi M, Balasubramaniam K," Simple capacitors to supercapacitors-an overview", International Journal of Electrochemical science Vol.3,2008.

[2] Mahapatra. S, Acharya. A, and Roy .S.G, "The role of nanomaterial for the design ofsupercapacitor,'Lat.Am.J.Phys.Educ.Vol.6, N03,Sept, 2012.

[3] Shao Quingguo, Jie Tang, and Yuexian Lin, "Carbon Nanotube spaced graphene aerogels with enhanced capacitance in aqueous and ionic liquid electrolytes", published by Elsevier B.V,2014.

[4] Ban Shuai, Jing Xie, and Zhou Hongjun, "Experimental and modeling study on charge storage / transfer mechanism of graphene based supercapacitors", published by Elsevier B.V, 15 June 2014.

[5] Rhet C.de Guzman, Jinho Yang, Mark Ming-Cheng Cheng and O. Salley Steven, "Effects of graphene and carbon coating modifications on electrochemical performance of silcon nanoparticle/graphene composite anode", published by Elsevier B.V,2014.

[6] Zheng Chao, Zhou Xufeng, Cao Hailing and Wang Guohua ,"Synthesis of porous graphene/activated carbon composite with high packing density and large specific surface area for supercapacitor electrode material", published by Elsevier B.V,2014.

[7] Wang Yaning, Chen Junchen,Cao Jianyun, Lin Yan,Zhou Yu,Ouyang Jia-Hu and Jia Dechang, "Graphene /carbon black hybrid film for flexible and high rate performance supercapacitor," published by Elsevier B.V,11 august 2014.

[8] Bello Abdulhakeem ,Barzegar Farshad and Momodu Damilola ,"Asymmetricsupercapacitor based on nanostructure graphene foam/polyvinyl alcohol/formaldehyde and activated carbon electrodes", published by Elsevier B.V ,22 september 2014.

[9] Jin Hong ,Wang Xiamin, and Gu Zhengrong , "A facile method for preparing nitrogen-doped graphene and its application in supercapacitors," published by Elsevier B.V,13 october 2014.

[10] Cai Xiaoyi, Lim Hua San and Poh Kok Chu, "High Performance asymmetric pseudocapacitor cell based on cobalt hydroxide/graphene and polypyrrole/graphene electrodes," published by Elsevier B.V,2014.

[11] Palacios Tomas, Allen Hsu, and Han Wang, 2010, "Applications of Graphene Devices in RF Communications," IEEE Communications Magazine, Vol. 0163.

[12] Cao Yang, Patricia C. Irwin and Younsi Karim , 2004,"Future of Nanodielectrics in the Electrical Power Industry", IEEE transactions on Dielectric and electrical Insulation, Vol. 11,Issue no. 5.

[13] Burges Tim, Chennupati Jagadish , 2008,"Nanoscale Materials: How Small is Big", Proceedings of IEEE, Vol.96, Issue No. 12.

[14] Krishnaswamy Dilip ,Amr Helmy and David Wentzloff 2010,"Applications of nanotechnology in communication", IEEE communication magazine.

[15] Fromille S and Phillips J, "Superdielectric Materials", Materials 2014, 7, 8197-8212; doi: 10.3390/ma7128197.

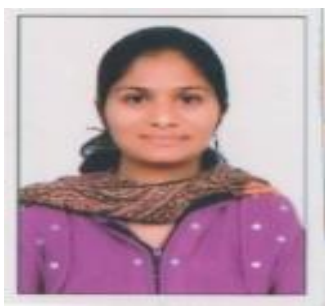

Vandana Sharma is an M.Tech Scholar from Ambala College Of Engineering, Ambala, Haryana, India. She has completed B. Tech (Hons.) in Electronics and Communication from Haryana Engineering College ,Jagadhri , India in 2011. She has completed Diploma (ECE) from Kalpana Chawla Govt. Polytechnic for Women, Ambala City under state board of technical education in 2008.Her Research interest includes Nanotechnology, Embedded Systems and Wireless Communication.

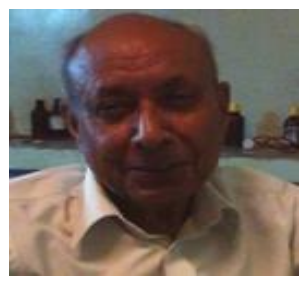

Mr. S.C. Sood is working as Research Associate in the field of nanotechnology in Ambala College Of Engineering and Applied Research, Ambala, Haryana, India( Electronics and communication department).He has completed B.Sc in 1965 and M.Sc (phy) with specialization in Electronics in 1970.He has 30 years experience in the field of fabrication of semiconductor power devices.

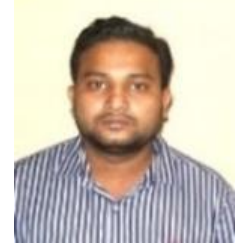

Er. Avesh Garg is presently working as Assistant Professor in Ambala College Of Engineering and Applied Research, Ambala, Haryana,India. $\mathrm{He}$ has Completed B. Tech(ECE) in 2009 and M. Tech (ECE) in 2011.He has 4 years experience in teaching and has keen interest in nanocomposites and nanoparticles. 